\title{
MOVILIDAD, APROVISIONAMIENTO Y USO DE OBSIDIANA EN EL PAYÉN, SUR DE LA PROVINCIA DE MENDOZA, ARGENTINA
}

\section{MOBILITY, PROCUREMENT AND USE OF OBSIDIAN IN EL PAYÉN, SOUTH OF THE PROVINCE OF MENDOZA, ARGENTINA}

\author{
Laura Salgán ${ }^{1}$, Adolfo Gil² y Gustavo Neme ${ }^{3}$ \\ 1 Museo de Historia Natural de San Rafael-ICES Malargüe, Rodríguez esq. Esquivel Aldao s/n 5613, Malargüe, \\ Mendoza. E-mail: mlaurasalgan@gmail.com \\ 2 CONICET - Museo de Historia Natural de San Rafael, Parque Mariano Moreno s/n 5600, San Rafael, Mendoza. \\ E-mail: adolfogil@arqueologiamendoza.com \\ 3 CONICET - Museo de Historia Natural de San Rafael, Parque Mariano Moreno s/n 5600, San Rafael, Mendoza. \\ E-mail: gustavoneme@arqueologiamendoza.com
}

Presentado el: 24/10/2012 - Aceptado 10/07/2013

\section{Resumen}

Las poblaciones humanas que ocuparon La Payunia en el Holoceno tardio, utilizaron obsidiana procedente de fuentes extra regionales con una direccionalidad de traslado predominante oeste-este. En el presente trabajo se analiza la distribución espacial de artefactos de obsidiana de procedencia geográfica conocida en el área El Payén. Se evalúan las posibles rutas de traslado, las estrategias de aprovisionamiento y la intensidad de uso de dicho recurso, sobre la base de indicadores tecnológicos y biogeográficos. Los resultados obtenidos plantean que si bien la direccionalidad de traslado de obsidiana coincide con la planteada para la región (oeste-este), su frecuencia en los sitios disminuye de sur a norte. Asimismo, los datos muestran diferencias en el número de fuentes representadas y en los modos de aprovisionamiento y uso de este recurso entre los sectores Centro, Sur y Norte de El Payén.

Palabras claves: tecnología lítica, obsidiana, cazadores recolectores, sur de Mendoza.

\footnotetext{
Abstract

Human populations that occupied The Payunia during the late Holocene, used obsidian from outside sources, with predominant east-west directionality. In this paper we analyze the spatial distribution of obsidian artifacts from known geographical origin in El Payén. We investigate the possible transport routes, procurement strategies and forms and intensity of use of that resource,
} 
from the base of technological and biogeography indicators. The results suggest that although the directionality of movement of obsidian matches that proposed for the region (west-east), its frequency decreases from south to north. The results also show differences in the number of sources represented and the procurement modes and use of this resource among Central, South and North sectors of El Payén.

Keywords: lithic technology, obsidian, hunter gatherer, south Mendoza.

\section{Introducción}

La distribución espacial de las materias primas líticas es una variable utilizada con frecuencia para estudiar la movilidad, los rangos de acción y los procesos de interacción entre las poblaciones humanas (Renfrew 1977; Meltzer 1989; Beck et al. 2002; Jones et al. 2012). En el Centro Occidental Argentino los primeros abordajes estuvieron orientados principalmente a la búsqueda e identificación geoquímica de las fuentes dentro de la región y a la caracterización de procedencia de muestras arqueológicas (Seelenfreud et al. 1996; Durán et al. 2004; De Francesco et al. 2006; Giesso et al. 2011). Luego, se abordaron tendencias temporales en el uso de las fuentes y su incorporación dentro de los circuitos de producción (Durán et al. 2004; Neme y Gil 2008; Barberena et al. 2011; Giesso et al. 2011; Cortegoso et al. 2012). Estos trabajos enfatizaron problemas de escala macro espacial, sin considerar el papel que jugaron los aspectos tecnológicos en los patrones encontrados. Estudios recientes en La Payunia, incorporan las características tecnológicas para explorar comparativamente el aprovisionamiento y uso de la obsidiana entre las áreas de El Nevado y El Payén (Salgán et al. 2012). En estos se propuso que en el área de El Nevado los artefactos de la fuente local y de acceso directo (El Peceño) se destacan por sobre una gran variedad de artefactos provenientes de fuentes distantes. En El Payén, por su parte, la materia prima más frecuente procede de la fuente no local cordillerana (Laguna El Maule), ubicada a una distancia lineal aproximada de $200 \mathrm{~km}$. A ésta siguen en frecuencia artefactos provenientes de la fuente no local más cercana Cerro Huenul. La escasez de artefactos procedentes de El Peceño en el área El Payén, sumado a la ausencia hasta el momento de artefactos procedentes de Laguna El Maule en el área El Nevado, fueron interpretados como resultado de diferencias en los circuitos de movilidad y en los rangos de acción de los grupos humanos (Salgán et al. 2012). En ambas áreas la obsidiana representa menos de $10 \%$ del total de materias primas utilizadas, sin embargo ocupa el segundo lugar en orden de frecuencia luego de las rocas silíceas de amplia disponibilidad local (Salgán et al. 2012; Salgán et al. 2013). En este trabajo se abordan las tendencias tecnológicas preliminares sobre el aprovisionamiento y modo de uso de la obsidiana, ajustando la escala de análisis al área El Payén. Para éste se tuvieron en cuenta los análisis de fluorescencia de rayos $\mathrm{X}$ (XRF) y de activación neutrónica (INAA) realizados sobre artefactos prevenientes de sitios arqueológicos del área y la información geoquímica disponible de las canteras de obsidiana del sur de Mendoza y norte de Neuquén (Barberena et al. 2011; Giesso et al. 2011; Cortegoso et al. 2012).

\section{Fuentes de obsidiana en el Centro Occidente Argentino}

La diversidad y procedencia de los artefactos de obsidiana en el sur de Mendoza ha sido un tema fuertemente abordado en los últimos veinte años (Durán et al. 2004; De Francesco et al. 2006; Barberena et al. 2011; Giesso et al. 2011; Salgán et al. 2012; Cortegoso et al. 2012). Hasta el momento se han identificado un total de siete fuentes de obsidiana, cinco geográficamente conocidas (Laguna del Diamante-Paramillos, Laguna El Maule, El Peceño, Las Cargas y Cerro 
Huenul) y dos desconocidas (provisoriamente denominadas: Desconocida y Desconocida A) (Giesso et al. 2011; Cortegoso et al. 2012) (Figura 1). De las fuentes conocidas, tres se encuentran en la cordillera andina por sobre los $2.500 \mathrm{msnm}$ (Laguna del Diamante, Las Cargas y Laguna El Maule) y son accesibles sólo en época estival (Seelenfreund et al. 1996; Durán et al. 2004; De Francesco et al. 2006; Giesso et al. 2011; Cortegoso et al. 2012) y, las dos restantes en el área

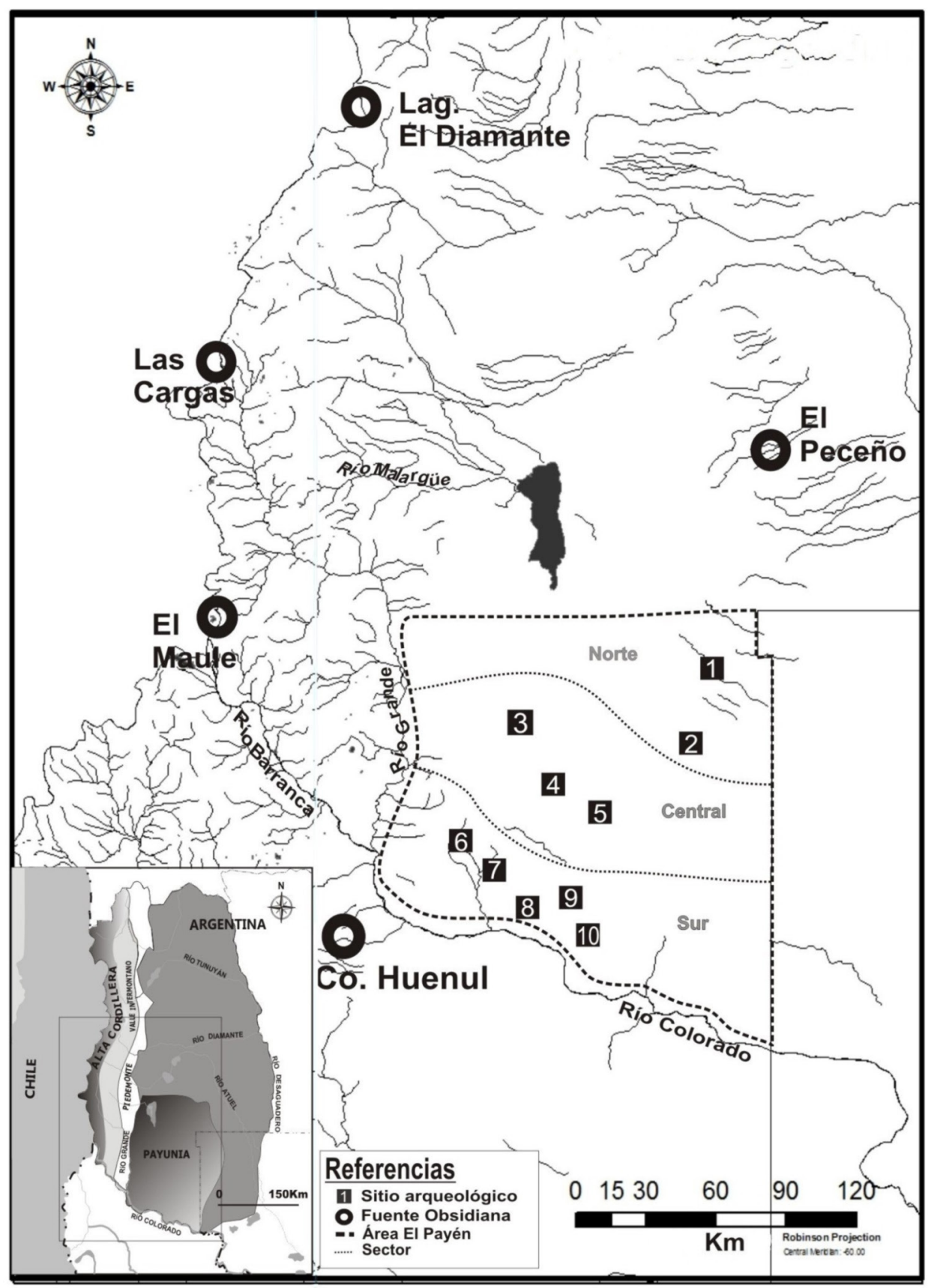

Figura 1. Ubicación de las fuentes de obsidiana y sitios muestreados en La Payunia. Referencias: Sector Norte: 1: Cueva Delerma; 2: La Peligrosa. Sector Central: 3: Puesto Mira; 4: Loma Alta; 5: ALPA-Este. Sector Sur: 6: Agua de Pérez; 7: Carmonina 1; 8: Rincón Blanco; 9: PRC Tren; 10: PRC. 
extra cordillerana (Cerro Huenul y El Peceño). Estas últimas están disponibles todo el año y son de fácil acceso (Durán et al. 2004; Barberena et al. 2011; Giesso et al. 2011; Cortegoso et al. 2012). Entre los artefactos arqueológicos muestreados en El Payén se detectó el uso de seis de las siete fuentes documentadas (Laguna El Maule, Las Cargas, El Peceño, Cerro Huenul, Desconocida y Desconocida A), con una distancia aproximada a las fuentes conocidas de entre 30 y $200 \mathrm{~km}$ lineales desde los sitios del área (Salgán et al. 2012) (Figura 1).

\section{Consideraciones teóricas y metodológicas}

La ubicación geográfica de las fuentes de recursos líticos y el trazado de distancias mínimas permiten estimar el transporte de objetos y/o la distancia de desplazamiento de los individuos (Renfrew 1977; Kuhn 1989; Ingbar 1994; Stern y Franco 2000). En este sentido, el uso de rocas de disponibilidad local (sensu Civalero y Franco 2003) es comúnmente considerado de acceso directo e indicador de rangos de acción (Binford 1979). En cambio, el aprovechamiento de rocas no locales o exóticas es interpretado o entendido como resultado de la interacción entre poblaciones y, por consiguiente, del intercambio de recursos (Meltzer 1989). En un trabajo previo y sobre la base de las estudios disponibles en la bibliografía arqueológica (Renfrew 1977; Binford 1979; Gould y Saggers 1985; Meltzer 1989; Bamforth 1991; Ingbar 1994; Franco 2004; Kuhn 2004; Charlin 2009), se generaron expectativas con el fin de explorar los modos de aprovisionamiento de obsidiana (Salgán et al. 2012). Estas se resumen en la tabla 1.

\begin{tabular}{|l|l|l|}
\hline Expectativas Arqueológicas & Acceso Directo & Acceso Indirecto \\
\hline $\begin{array}{l}\text { Frecuencia de materia prima en el } \\
\text { conjunto }\end{array}$ & Alta & Baja \\
\hline $\begin{array}{l}\text { Representación de estadios de } \\
\text { manufactura }\end{array}$ & Todas las etapas & Predominio de etapas finales \\
\hline Clases artefactuales & Amplio & Restringido \\
\hline Tamaño de los artefactos & Variable & Muy pequeños -pequeños \\
\hline Porcentaje de Corteza & Alto & Bajo \\
\hline Núcleos & $\begin{array}{l}\text { Variabilidad en grado de } \\
\text { reducción. Predominio formas } \\
\text { amorfas. }\end{array}$ & Ausentes o muy explotados. \\
\hline Desechos de Talla & $\begin{array}{l}\text { Alta variabilidad y frecuencia, } \\
\text { todas las etapas de reducción } \\
\text { aunque prevalecen las finales. }\end{array}$ & $\begin{array}{l}\text { Baja variabilidad, prevalecen } \\
\text { reactivación de artefactos. }\end{array}$ \\
\hline Instrumentos & $\begin{array}{l}\text { Alta variabilidad de tipos } \\
\text { artefactuales. Artefactos con } \\
\text { corteza. }\end{array}$ & $\begin{array}{l}\text { Alta frecuencia en relación a } \\
\text { otras clases artefactuales. } \\
\text { Baja variabilidad en tipos, sin } \\
\text { corteza. }\end{array}$ \\
\hline
\end{tabular}

Tabla 1. Expectativas arqueológicas de los conjuntos artefactuales según el modo de aprovisionamiento (Salgán et al. 2012).

En este aporte se aborda desde una perspectiva tecnológica, la distribución de artefactos de obsidiana de procedencia geoquímica conocida del área El Payén. Con fines heurísticos se propuso subdividir el área en tres sectores: Norte, Central y Sur (Figura 1). Estos se corresponden con características geológicas y de disponibilidad de recursos hídricos diferenciales (Salgán 2012). El análisis intenta dar cuenta del aprovisionamiento, secuencia 
de reducción e intensidad de uso de los artefactos de obsidiana, con el fin de avanzar en entender los patrones de movilidad y rangos de acción de los grupos humanos que ocuparon el área durante el Holoceno tardío.

Se procesaron artefactos de diez sitios arqueológicos, en su mayoría a cielo abierto, en los que se recuperó un total de 278 artefactos de obsidiana, integrados por desechos de talla $(86 \%, n: 239)$, instrumentos (13\%, n: 36) y núcleos (1\%, n: 3). El muestreo de los artefactos se llevó a cabo en el marco de un proyecto mayor, cuyo objetivo fue caracterizar la variabilidad macroscópica en las obsidianas (Giesso et al. 2011). Asimismo, y debido a que gran parte de las muestras fueron analizadas por método de XRF (97\%), el muestreo implico la selección de artefactos de tamaño (mayor a 3, sensu Aschero 1983) y espesor (mayor a $5 \mathrm{~mm}$ ) particulares (Glascock 2010). Esto redujo el número de muestras potenciales de análisis a 120 artefactos (83 desechos de talla, 36 instrumentos y 3 núcleos), de los que se procesaron 98 (66 desechos de talla, 29 instrumentos y 3 núcleos).

Los estudios geoquímicos fueron realizados en el Missouri University Research Reactor (MURR), siguiendo la metodología para INAA descripta en Durán et al. (2004) y para XRF en Giesso et al. (2011). La información geoquímica de los artefactos corresponde a la publicada en Giesso et al. (2011) y Salgán (2012). Respecto a los sitios arqueológicos considerados, tres son estratigráficos y de ellos procede el $10 \%$ de los artefactos analizados. Los siete restantes son superficiales, concentrando el 90\% de la muestra (Figura 1). Dos de los sitios estratigráficos se encuentran en el sector Norte, en la localidad La Peligrosa (La Peligrosa 2, n: 8 y Cueva Delerma, n: 1) y uno en el sector Sur, en la localidad Carmonina (Carmonina 1, n: 1). Las prospecciones superficiales se realizaron en el sector Central (Puesto Mira, n: 3; Loma Alta, n: 26 y; Altiplanicie Este, n: 17) y en el sector Sur (APE, n: 3; RC, n: 11 y; Rincón Blanco, n: 28).

Los atributos tecnológicos considerados son: 1) frecuencia de artefactos según fuente de procedencia; 2) clase artefactual: instrumentos (formatizados: unifaciales y bifaciales; artefactos sin formatización con rastros complementarios), núcleos y desechos de talla (Aschero 1975, 1983; Aschero y Hocsman 2004); 3) corteza (rangos de $0 \%, 50 \%$ y $100 \%$ de corteza en su cara dorsal); 4 ) modo de descarte de los instrumentos, entero o fracturado; 5) tamaño (sensu Aschero 1975, 1983); 6) módulo ancho/espesor; 7) etapa de reducción (Salgán et al. 2012) y; 8) densidad de negativos de lascados (o extracciones previas) en la cara dorsal de los desechos de talla (Ingbar et al. 1989). En el análisis tecnológico de los artefactos se priorizó el tratamiento de las muestras por sector (Norte, Central y Sur).

Cabe aclarar que los análisis tecno-morfológicos de las piezas se profundizaron a posteriori del análisis geoquímico, razón por la cual tres de las muestras analizadas por medio de métodos destructivos (INAA), sólo pudieron ser diferenciadas a nivel de clase artefactual (3\%).

\section{Resultados}

En el área El Payén el mayor número de artefactos procede de la cantera Laguna El Maule (65\%, n: 64), seguida por Cerro Huenul (25\%, n: 15), Las Cargas (10\%, n: 10), Desconocida (5\%, n: 5), El Peceño (3\%, n: 3) y Desconocida-A (1\%, n: 1) (Tabla 2). Sin embargo, su distribución en el área no es homogénea. En el sector Norte el primer lugar en orden de frecuencia de 
artefactos procede de la fuente Laguna El Maule (56\%, n: 5), seguido por El Peceño (33\%, n: 3) y Desconocida-A $(11 \%, n: 1)$. Las fuentes conocidas se encuentran en el rango de distancia de las rocas no locales (sensu Civalero y Franco 2003) y en ambos casos superan los $100 \mathrm{~km}$ de distancia lineal (Tabla 2). En el sector Central, son frecuentes los artefactos de Laguna El Maule (48\%, n: 22), seguido por Las Cargas (22\%, n: 10), Cerro Huenul (22\%, n: 10) y Desconocida $(9 \%, \mathrm{n}: 4)$. Las tres fuentes conocidas son consideradas no locales, las dos primeras provienen de distancias superiores a los $100 \mathrm{~km}$ lineales, mientras que Cerro Huenul que se encuentra entre los 55 y $65 \mathrm{~km}$ lineales. En el sector Sur, Laguna El Maule $(86 \%, n: 37)$ ocupa el primer lugar en frecuencia de artefactos, seguida por Cerro Huenul $(12 \%$, n: 5$)$ y Desconocida $(2 \%, n$ : 1$)$. Laguna El Maule es considerada no local, mientras que Cerro Huenul se encuentra en el rango de distancia de las rocas locales (Tabla 2).

La tabla 3 muestra las clases artefactuales según su fuente de procedencia y sector. En el Norte, la obsidiana Laguna El Maule se registra como desechos de talla e instrumentos. Las otras fuentes como desechos de talla. En el Central, los artefactos de Laguna El Maule, Cerro Huenul y Las Cargas corresponden a desechos de talla e instrumentos, mientras que entre las Desconocidas se registran las tres clases artefactuales (instrumentos, desechos de

\begin{tabular}{|c|c|c|c|c|c|c|c|c|c|c|c|c|c|c|c|c|c|c|c|c|c|c|c|c|c|c|c|c|c|c|c|}
\hline \multirow{3}{*}{ Fuentes } & \multicolumn{6}{|c|}{ Norte } & \multicolumn{8}{|c|}{ Central } & \multicolumn{17}{|c|}{ Sur } \\
\hline & \multicolumn{3}{|c|}{ La Peligrosa } & \multicolumn{3}{|c|}{$\begin{array}{c}\text { Cueva } \\
\text { Delerma } \\
\end{array}$} & \multicolumn{3}{|c|}{ ALPA Este } & \multicolumn{2}{|c|}{ Loma Alta } & \multicolumn{3}{|c|}{ Puesto Mira } & \multicolumn{3}{|c|}{ PRC } & \multicolumn{4}{|c|}{ PRC Tren } & \multicolumn{3}{|c|}{$\begin{array}{l}\text { Rincón } \\
\text { Blanco }\end{array}$} & \multicolumn{3}{|c|}{ APE } & & \multicolumn{3}{|c|}{ CAR-1 } \\
\hline & $D$ & DT & \begin{tabular}{l|l} 
& 1 \\
\end{tabular} & $D$ & DT & $\mathrm{N} / \mathrm{I}$ & $D$ & DT & \begin{tabular}{|l|l|}
$N$ & 1 \\
\end{tabular} & \begin{tabular}{ll|l}
$D$ & $D T$ & $D$ \\
\end{tabular} & $\begin{array}{lll}N & 1 \\
\end{array}$ & $D$ & \begin{tabular}{|l|l|l} 
DT & N \\
\end{tabular} & $\begin{array}{lll}N & 1 \\
\end{array}$ & D & DT & \begin{tabular}{l|l}
$\mathrm{N}$ & 1 \\
\end{tabular} & $D$ & DT & \begin{tabular}{|l|l|}
$N$ \\
\end{tabular} & 1 & \begin{tabular}{l|l}
0 & 0 \\
\end{tabular} & \begin{tabular}{l|l}
$\mathrm{DT}$ & $\mathrm{N}$
\end{tabular} & $\sqrt{1}$ & $D$ & DT & \begin{tabular}{|l|l|l|}
$N$ & 1 \\
\end{tabular} & D & $D$ D & $\mathrm{DT}$ & $\begin{array}{lll}N & 1 \\
\end{array}$ \\
\hline El Peceño & 130 & 3 & & 100 & . & & 155 & & - & 160 & & 145 & - & & 210 & $\therefore$ & - & 200 & $=$ & . & $\therefore$ & 200 & - & 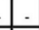 & 180 & - & - & 200 & 07 & - & $-1-$ \\
\hline Las Cargas & 20 & & . & 205 & . & & 180 & . & . & \begin{tabular}{|l|l|}
180 & 6 \\
\end{tabular} & 3 & 175 & . & 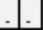 & 250 & . & . & 230 & . & . & 23 & 235 & . & . & 200 & . & . & 230 & 30 & - & -5 \\
\hline L. El Maule & 180 & . & 4 & 170 & 1 & & 115 & 4 & 2 & \begin{tabular}{|l|l}
120 & 14 \\
\end{tabular} & 2 & 112 & . & & 180 & 4 &. & 160 & 3 & - & 116 & 165 & 13 & 12 & 125 & 1 & & .15 & 51 & 1 & \\
\hline Cerro Huenul & 125 & & & 140 & - & & 55 & 7 & 1 & \begin{tabular}{|l|l|}
55 & - \\
\end{tabular} & & 62 & 1 & 1 & 80 & & 1 & 60 & 1 & . & .6 & \begin{tabular}{l|l}
65 \\
\end{tabular} & - & 1 & 30 & 1 & & \begin{tabular}{l|l}
1 & 50 \\
\end{tabular} & 50 & - & -5 \\
\hline Desconocida-A & i? & . & 1 & i? & . & & i? &. & .5. & $6 ?$ & . & ?? & .5. &. & i? & - & - & ?? & - & - & - & i? & - & - & $6 ?$ & - & - & $-4 ?$ & $?$ & - & - \\
\hline Desconocida & $?$ & - & - & i? & . & & .? & 1 & $\begin{array}{ll}.1 \\
\end{array}$ & $2 ?$ & & i? & - & 1 & i? & & . & i? & \begin{tabular}{|l|}
- \\
\end{tabular} & . & - & i? & & & i? & 2 & & 1 i? & $?$ & & \\
\hline Total Sitio & & 8 & & & 1 & & & 17 & & 26 & & & 3 & & & 6 & & & 5 & & & & 28 & & & 3 & & & & 1 & \\
\hline Total Sector & & & & 9 & & & & & & 46 & & & & & & & & & & & & & 43 & & & & & & & & \\
\hline
\end{tabular}

Tabla 2. Frecuencia de obsidiana por categoría tecnológica y fuente de procedencia en relación a los sitios y distancia a las fuentes. Referencias: D: Distancia aproximada en km; DT: Desechos de talla; N: Núcleos; I: Instrumentos.

talla y núcleos). En el Sur, por su parte, Laguna El Maule se presenta en las tres clases de artefactos, es decir, como desechos de talla, instrumentos y núcleos. Esto también se observa, pero en menor frecuencia, en artefactos confeccionados sobre obsidiana Cerro Huenul, mientras que en Desconocida sólo se registran desechos de talla.

En cuanto a la proporción de corteza en artefactos enteros, los porcentajes más altos de corteza se registran en el sector Central y Sur entre los ejemplares fabricados sobre obsidiana Cerro Huenul, mientras que los manufacturados sobre obsidiana de otras fuentes no presentan corteza. Tendencia que puede responder al modo en que se presenta la materia prima en la fuente (Durán et al. 2004; Barberena et al. 2011; Salgán et al. 2012).

En la tabla 4 se detalla el estado de fragmentación de los instrumentos y su frecuencia por fuente de procedencia. En el sector Norte y Central los instrumentos se encuentran en su mayoría fracturados, mientras que en el Sur se registra una mayor frecuencia de instrumentos enteros por sobre los fracturados. En el sector Norte, los dos instrumentos registrados son de obsidiana Laguna El Maule y corresponden al grupo tipológico de las puntas de proyectil. Ambas tienen el ápice fracturado y una de ellas presenta lascados de reactivación. En el Central, los instrumentos más frecuentes son los de formatización bifacial 


\begin{tabular}{|c|c|c|c|c|c|c|c|}
\hline & Clase Artefacto & Co Huenul & El Peceño & L. El Maule & Las Cargas & Desc & Desc-A \\
\hline \multirow{3}{*}{ Norte } & AF Bifacial & - & - & $2(40 \%)$ & - & - & - \\
\hline & DT & - & $3(100 \%)$ & $3(60 \%)$ & - & - & $1(100 \%)$ \\
\hline & N Total & - & $3(33 \%)$ & $5(55 \%)$ & - & - & $1(12 \%)$ \\
\hline \multirow{5}{*}{ Central } & AF Bifacial & $1(10 \%)$ & - & $3(14 \%)$ & $3(30 \%)$ & - & - \\
\hline & AF Unifacial & $1(10 \%)$ & - & $1(5 \%)$ & $1(10 \%)$ & $1(25 \%)$ & - \\
\hline & DT & $8(80 \%)$ & - & $18(82 \%)$ & $6(60 \%)$ & $2(50 \%)$ & - \\
\hline & Núcleo & - & - & & - & $1(25 \%)$ & - \\
\hline & N Total & $10(22 \%)$ & - & $22(48 \%)$ & $10(22 \%)$ & $4(9 \%)$ & - \\
\hline \multirow{6}{*}{ Sur } & AF Bifacial & $1(20 \%)$ & - & $6(16 \%)$ & - & - & - \\
\hline & AF Unifacial & - & - & $6(16 \%)$ & - & - & - \\
\hline & ASF RC & $1(20 \%)$ & - & $2(5 \%)$ & - & - & - \\
\hline & DT & $2(40 \%)$ & - & $22(59 \%)$ & - & $1(100 \%)$ & - \\
\hline & Núcleo & $1(20 \%)$ & - & $1(3 \%)$ & - & - & - \\
\hline & N Total & $5(12 \%)$ & - & $37(86 \%)$ & - & $1(2 \%)$ & - \\
\hline
\end{tabular}

Tabla 3. Representación de cada fuente por clase tipológica y por sector. Referencia: AF Bifacial: Artefacto de Formatización Bifacial; AF Unifacial: Artefacto de Formatización Unifacial; ASF RC: Artefacto Sin Formatización con Rastros Complementarios; DT: Desecho de Talla; L. El Maule: Laguna El Maule; Desc: Desconocida.

$(64 \%, n=7)$, seguidos por los unifaciales $(36 \%, n: 4)$, todos confeccionados sobre lasca. En este sector, la obsidiana Laguna El Maule, está representada por un cuchillo de filo lateral de retoque bifacial entero, dos puntas de proyectil (una fracturada) y un artefacto unifacial de retoque en bisel oblicuo entero. En obsidiana procedente de Las Cargas se registraron dos puntas de proyectil recuperadas en estado fracturado y dos artefactos no diferenciados por fractura, uno de formatización bifacial y otro unifacial. Asimismo, la procedente de Cerro Huenul está representada por dos artefactos formatizados no diferenciados por fractura (Tabla 3 y 4 ).

En el sector Sur, los artefactos de formatización bifacial (43\%, n: 6) y unifacial (43\%, n: 6) están representados en igual proporción, seguidos por tres artefactos sin formatización con rastros complementarios (14\%) (Tabla 3). El 81\% de los instrumentos se encuentran enteros (n: 13) y el $19 \%$ restante fracturado (n: 3), todos confeccionados sobre lasca. Se han identificado las fuentes: Laguna El Maule (88\%, n: 14) y Cerro Huenul (12\%, n: 2). Sobre la primera se recuperaron seis artefactos bifaciales (dos artefactos de filo lateral corto, dos con filo convergente en ápice romo y dos puntas de proyectil, estas últimas fracturadas) y seis unifaciales (tres filos laterales cortos enteros, un posible perforador con reserva de corteza en toda su cara dorsal fracturado y dos artefactos con rastros complementarios enteros). En obsidiana de Cerro Huenul se recuperó una punta de proyectil y un artefacto con rastros complementarios, ambos enteros (Tabla 4).

En cuanto a los módulos de medida en instrumentos enteros hay una tendencia a módulos espesos y muy espesos (sensu Aschero 1975, 1983) en los sectores Central y Sur sobre obsidiana Laguna El Maule (Central: 75\%, n: 3; Sur: 95\%, n: 11) y en el Sur sobre obsidiana Cerro Huenul (100\%, n: 2) (Salgán 2012). La proporción de módulos muy espesos puede indicar el descarte de artefactos con potencial de reactivación y/o mantenimiento (Franco 2004; Charlin 2007). En lo referido al tamaño relativo de los instrumentos enteros 


\begin{tabular}{|c|c|c|c|c|c|c|}
\hline Sector & Estado & Cerro HuenuLag. El Maule & Las Cargas & Desc & N \\
\hline \multirow{4}{*}{ Norte } & Entero & - & 2 & - & - & $2(100 \%)$ \\
\cline { 2 - 7 } & Fracturado & - & - & - & - & - \\
\hline \multirow{3}{*}{ Central } & Entero & - & 3 & - & 1 & $4(36 \%)$ \\
\cline { 2 - 7 } & Fracturado & 2 & 1 & 4 & - & $7(64 \%)$ \\
\hline \multirow{3}{*}{ Sur } & Entero & 2 & 11 & - & - & $13(81 \%)$ \\
\cline { 2 - 7 } & Fracturado & - & 3 & - & - & $3(19 \%)$ \\
\hline
\end{tabular}

Tabla 4. Estado de fragmentación de artefactos formatizados (Bifaciales y Unifaciales) y no formatizados con filo, puntas y/o superficies con rastros complementarios en función de los sectores y variedades de obsidiana. Referencia: Desc: Desconocida

en los sectores Central y Sur, predominan los tamaños grandes (grandísimo, muy grande y grande; sensu Aschero 1975, 1983) en obsidiana Laguna El Maule (Central: 100\%, n: 3; Sur 100\%, n: 11), Cerro Huenul (Sur: 50\%, n: 2) y Desconocida (Central: 100\%, n: 1). Le sigue el tamaño mediano pequeño en el sector Sur (Cerro Huenul: 50\%, n: 2).

En cuanto a desechos de talla analizados geoquímicamente, en el sector Norte se registraron siete lascas internas (lascas angulares) correspondientes a El Peceño (n: 3), Laguna El Maule (n:3) y Desconocida-A (n: 1), vinculadas a etapas intermedias de manufactura (Tabla 5). En los sectores Central (n: 34) y Sur (n: 25), en cambio, están presentes todas las etapas del proceso de manufactura. En obsidiana Cerro Huenul se registran desechos asociados a las primeras etapas de manufactura (lascas primarias y secundarias) en el sector Sur, mientras que en el sector Central se encuentran representados todos los estadios de reducción. En obsidiana Las Cargas (sector Central) se reconocen lascas internas y de mantenimiento de filos (lascas de reactivación). En la variedad Desconocida sólo se registran lascas internas.

Al considerar el grado de reducción de las distintas variedades de obsidiana por medio del cálculo del índice de corteza (Ericson 1984) y de intensidad de reducción de las materias primas (Ingbar et al. 1989; Ingbar 1994) (Tablas 6 y 7), se observa que en los sectores Central y Sur el mayor índice de corteza se registra en obsidiana Cerro Huenul, seguido por la obsidiana Laguna El Maule, sin registro de valores de corteza en las demás variedades. Respecto a la intensidad de reducción, los valores medios mayores se registran en el sector Central en obsidiana Desconocida y en el sector Sur en obsidiana Laguna El Maule. Si comparamos las tendencias registradas, observamos que en obsidiana Cerro Huenul y Laguna El Maule el índice de corteza es mayor en el sector Sur respecto al Central (para el cálculo de ambos índices se excluyeron las lascas de reactivación de filo y en el caso del índice de corteza sólo se consideraron las lascas enteras) (Tabla 6). Sin embargo, se observan diferencias entre ambos sectores en la intensidad de reducción de desechos de talla de la obsidiana Cerro Huenul, siendo mayor en el sector Central que en el Sur. No obstante, en obsidiana Laguna El Maule el valor medio de reducción es mayor en el Sur (Tabla 7). Lo observado podría indicar que la obsidiana Laguna El Maule habría ingresado en el sector Sur con mayor reserva de corteza que en el Central, dado que en el Sur son más frecuentes las lascas primarias y secundarias y los valores mínimos de intensidad de reducción son igual a cero (Tabla 7).

Asimismo, en los sectores Central y Sur existe alta variabilidad en la relación entre densidad de extracciones y rangos de distancia (Figura 2, Tabla 7). En el sector Central la mayor variabilidad se presenta en obsidiana Cerro Huenul (rango de distancia entre $41 \mathrm{y}$ $80 \mathrm{~km}$ ) y Las Cargas (rango de distancia entre 161 y $200 \mathrm{~km}$ ), con la presencia de todas las 
etapas de manufactura en la primera. En Las Cargas solo se registran lascas internas con distinta intensidad de lascado además de una lascas de reactivación de filo. En artefactos Laguna El Maule (rango entre 81 y $120 \mathrm{~km}$ ) se observa menor variabilidad en la intensidad de extracciones, pero con valores extremos (como lascas primarias y desechos con alta intensidad de lascado). Sin embargo, las diferencias de varianza en la intensidad de lascado de las distintas variedades de obsidianas en el sector Central no son significativas (ANOVA, $p=0,722 ; d f .=3)$. La distancia a la fuente no parece ser un factor que esté influyendo en la intensidad de lascados de los artefactos en este sector. En el Sur, la obsidiana Laguna El Maule (rango de distancia entre 161 y $200 \mathrm{~km}$ ) presenta una variabilidad semejante a la observada en el sector Central. Si bien posee un valor medio de densidad de extracción mayor, este no resulta significativo $(t=0,35 ; \mathrm{p}=0,72)$. La obsidiana Cerro Huenul sólo se presenta como lascas externas, con escasa variabilidad. Al comparar la intensidad de extracciones en artefactos de obsidiana entre sectores, las diferencias observadas no resultan significativas (ANOVA, $p=0,89 ; d f .=5)$. La distancia de procedencia de la materia prima, por lo tanto, no afecta de manera significativa su intensidad de uso.

En cuanto a los núcleos recuperados (n: 3), uno corresponde al sector Central (34\%) y dos al sector Sur (66\%). El primero fue confeccionado sobre obsidiana de fuente desconocida. Se encuentra fragmentado, está cubierto por una gruesa pátina y su tamaño estimado es muy grande. Presenta una densidad de extracciones baja $(x=0,493 \mathrm{e} / \mathrm{mm} 3)$ y su forma base no pudo ser determinada. Los núcleos recuperados en el sector Sur provienen de las fuentes Laguna El Maule y Cerro Huenul. El primero es piramidal y el segundo amorfo y ambos

\begin{tabular}{|c|c|c|c|c|c|c|c|}
\hline \multirow{3}{*}{ Sector } & DT & Co Huenul & El Peceño & $\begin{array}{c}\text { Laguna El } \\
\text { Maule }\end{array}$ & Las Cargas & Desc & Desc-A \\
\hline Norte & LAN & - & $3(100 \%)$ & $3(100 \%)$ & - & - & $1(100 \%)$ \\
\hline \multirow{5}{*}{ Central } & LPR & $2(29 \%)$ & - & - & - & - & - \\
\cline { 2 - 8 } & LSEC & - & - & $1(6 \%)$ & - & - & - \\
\cline { 2 - 8 } & LAN & $1(14 \%)$ & - & $8(44 \%)$ & $3(50 \%)$ & $1(50 \%)$ & - \\
\cline { 2 - 8 } & LAR & $1(14 \%)$ & - & $4(22 \%)$ & $2(34 \%)$ & $1(50 \%)$ & - \\
\cline { 2 - 8 } & LAB & $1(14 \%)$ & - & - & - & - & - \\
\cline { 2 - 8 } & PL & - & - & $2(11 \%)$ & - & - & - \\
\cline { 2 - 8 } & LR & - & - & $3(17 \%)$ & $1(16 \%)$ & - & - \\
\cline { 2 - 8 } & LN & $2(29 \%)$ & - & - & - & - & - \\
\hline & LPR & & - & $1(5 \%)$ & - & - & - \\
\cline { 2 - 8 } & LSEC & $1(50 \%)$ & - & $4(20 \%)$ & - & - & - \\
\cline { 2 - 8 } & LAN & - & - & $7(32 \%)$ & - & $1(100 \%)$ & - \\
\cline { 2 - 8 } & LAR & - & - & $6(27 \%)$ & - & - & - \\
\cline { 2 - 8 } & LR & - & - & $1(5 \%)$ & - & - & - \\
\cline { 2 - 8 } & LN & $1(50 \%)$ & - & $2(9 \%)$ & - & - & - \\
\cline { 2 - 8 } & LBIP & - & - & $1(5 \%)$ & - & - & - \\
\hline
\end{tabular}

Tabla 5. Desechos de talla de cada área; frecuencia relativa de cada fuente. Referencias: LPR: lasca primaria; LSEC: lasca secundaria; LAN: lasca angular; LAR: lasca de arista; LAB: lasca de adelgazamiento bifacial; LR: lasca de reactivación de instrumentos; LN: lasca nodular; LBIP: lasca bipolar. 


\begin{tabular}{|c|c|c|c|c|c|}
\hline Sector & Fuente & N & LPR & LSEC & Índice corteza \\
\hline \multirow{7}{*}{ Central } & Co Huenul & 2 & 1 & - & 0,5 \\
\cline { 2 - 6 } & L. El Maule & 13 & - & 1 & 0,07 \\
\cline { 2 - 6 } & Las Cargas & 1 & - & - & - \\
\cline { 2 - 6 } & Desconocida & 1 & - & - & - \\
\hline \multirow{4}{*}{ Sur } & Co Huenul & 2 & - & 1 & 0,5 \\
\cline { 2 - 6 } & L. El Maule & 12 & 1 & 3 & 0,33 \\
\cline { 2 - 6 } & Desconocida & 1 & - & - & - \\
\hline
\end{tabular}

Tabla 6. Índice de corteza de las distintas fuentes en cada sector; para el cálculo de este índice no se consideran las lascas fracturadas y las de reactivación de filo (sensu Ericson 1984).

\begin{tabular}{|c|c|c|c|c|c|c|c|c|c|}
\hline \multirow{2}{*}{ Sector } & Fuente & $\mathbf{N}$ & Media & Mediana & $\begin{array}{c}\text { Desvío } \\
\text { Estándar }\end{array}$ & Mín & Máx & Rango & $\begin{array}{c}\text { Rango } \\
\text { Distancia }\end{array}$ \\
\hline \multirow{4}{*}{ Central } & Co Huenul & 8 & 0,511 & 0,351 & 0,621 & 0 & 1,71 & 1,71 & $41-80 \mathrm{~km}$ \\
\cline { 2 - 11 } & L. El Maule & 15 & 0,639 & 0,528 & 0,51 & 0,181 & 2,08 & 1,89 & $111-140 \mathrm{~km}$ \\
\cline { 2 - 11 } & Las Cargas & 5 & 0,819 & 0,932 & 0,432 & 0,32 & 1,28 & 0,96 & $171-200 \mathrm{~km}$ \\
\cline { 2 - 11 } & Desc & 2 & 0,847 & - & - & 0,544 & 1,15 & 0,6 & - \\
\hline \multirow{2}{*}{ Sur } & Co Huenul & 2 & 0,375 & - & - & 0,104 & 0,64 & 0,54 & $30-80 \mathrm{~km}$ \\
\cline { 2 - 11 } & L. El Maule & 21 & 0,677 & 0,462 & 0,683 & 0 & 2,48 & 2,48 & $171-200 \mathrm{~km}$ \\
\hline
\end{tabular}

Tabla 7. Estadística descriptiva de la densidad de extracciones previas en la cara dorsal de los desechos de talla de las distintas fuentes en cada sector.

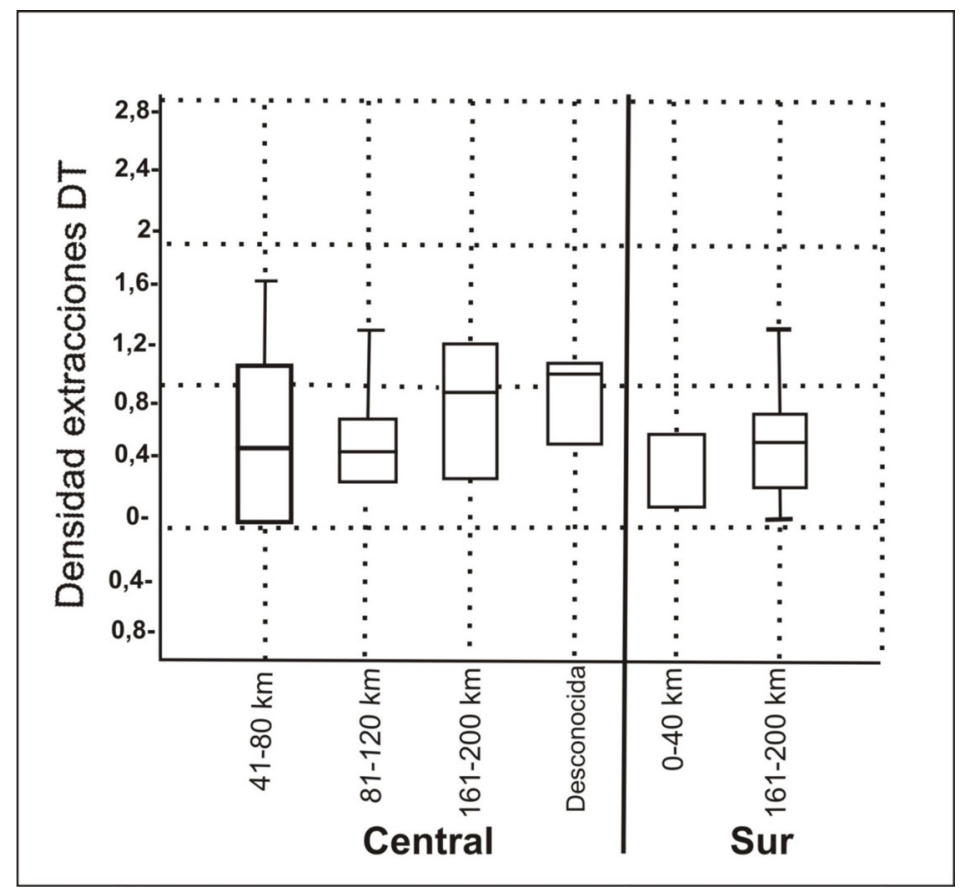

Figura 2. Frecuencia de densidad de extracciones en sectores Central y Sur, según distancia estimada en $\mathrm{km}$ a la fuente. 
se encuentran agotados. La densidad de extracciones o número de negativos de lascado es mayor en el núcleo de Laguna El Maule $(x=1,66$ e/mm3) y menor en el caso de Cerro Huenul $(x=1,588 \mathrm{e} / \mathrm{mm} 3)$. Sólo la pieza procedente de Cerro Huenul presenta remanentes de corteza $(25 \%)$.

\section{Tendencias geoquímicas y tecnológicas de las obsidianas de sitios arqueológicos del Área El Payén}

Los resultados hasta aquí detallados muestran diferencias significativas en cuanto a diversidad de fuentes de obsidiana documentadas, etapas de la secuencia de reducción representadas e intensidad de reducción, entre el sector Norte por un lado y, Central y Sur por otro. Si bien el número de muestras procesadas es bajo, el mismo representa más del $80 \%$ de los artefactos factibles de ser procesados por métodos geoquímicos. En los tres sectores la fuente cordillerana Laguna El Maule es la más representada -distante de la región donde se emplazan los sitios entre 120 y $200 \mathrm{~km}-$, aunque se destaca que la importancia de esta variedad en los conjuntos disminuye de sur a norte. Le sigue en frecuencia la fuente Cerro Huenul, distante entre 30 y $80 \mathrm{~km}$ de los sitios y ausente en el sector Norte. Respecto a la diversidad de fuentes de obsidiana representadas en cada sector, el Central posee los valores de diversidad más altos, en relación a los sectores Norte y Sur. En el Central los artefactos procedentes de las fuentes Cerro Huenul y Laguna El Maule presentan alta variabilidad de clases tipológicas y están representadas todas las etapas de reducción de artefactos. La obsidiana Las Cargas sólo se encuentra en este sector como desechos de talla correspondientes a los estadios finales de reducción e instrumentos fracturados. Además, en este sector se registra la frecuencia más alta de obsidianas con señal química no asignada o Desconocida. Esta posee alta variación de clases tipológicas (instrumentos, desechos de talla y núcleos), nula presencia de corteza y el descarte de los instrumentos se da como enteros.

En el sector Norte, por su parte, se registran tres variedades de obsidiana (El Peceño, Laguna El Maule y Desconocida-A). La obsidiana Laguna El Maule es la que presenta mayor variación de clases artefactuales (instrumentos y desechos de talla) mientras que El Peceño sólo se registra como desechos de talla. Es de destacar que este sector es el único donde se registra la señal química El Peceño y Desconocida-A. Esta última presente como lasca interna. Finalmente en el sector Sur, se registran dos fuentes de obsidiana conocidas (Laguna El Maule y Cerro Huenul) y una Desconocida. La obsidiana Laguna El Maule es la única que exhibe todas las etapas de manufactura, acompañada de alta variación de clases artefactuales. Entre los desechos de talla de obsidiana Cerro Huenul prevalecen las lascas externas correspondientes a las primeras etapas de manufactura. La presencia de corteza en artefactos Laguna El Maule se da en todas las clases artefactuales, con excepción de los núcleos, los que se encuentran agotados y con alta intensidad de lascados. En obsidiana Cerro Huenul se registran todas las clases tipológicas, pero en muy baja frecuencia, a pesar de su proximidad espacial. También las Desconocidas poseen baja frecuencia y sólo se registran como lascas internas.

Dada la ausencia de fuentes de obsidiana en El Payén, cabría esperar el registro frecuente de las fuentes distantes más próximas, quizás como resultado de la interacción entre grupos y/o la transferencia de bienes, así como la presencia esporádica de las fuentes más lejanas, con índices bajos de corteza y prevalencia de etapas finales de reducción. Se espera un acceso indirecto a esta materia prima, donde la clase tipológica más representada sea la de instrumentos y desechos de talla de reactivación de filos (Tabla 1). 
En base a las expectativas tecnológicas planteadas, la evidencia recuperada señala que la obsidiana de mayor frecuencia Laguna El Maule habría ingresado a la región en forma de núcleos y/o formas base con reserva de corteza. La variabilidad y frecuencia de desechos de talla permite sostener la manufactura de instrumental in situ, al menos en los sectores Central y Sur donde presenta altos valores de intensidad de reducción. En cuanto a los instrumentos, la mayor variabilidad de tipos se registra en sector Sur, seguido por el Central y, sólo presente como puntas de proyectil en el Norte. Dado el tamaño del instrumental y las formas base utilizadas, la búsqueda de soportes adecuados para la manufactura de puntas de proyectil parece ser el eje direccional del procesamiento de esta materia prima. En el sector Central, la obsidiana Laguna El Maule exhibe un bajo índice de corteza y no se registran núcleos, lo que podría indicar el ingreso de formas base o núcleos descortezados. En el sector Norte, por su parte, la presencia de lascas internas y de un instrumento con posible reactivación de filo, permite sostener de forma preliminar el ingreso al sector de instrumentos formatizados y/o formas base en avanzado estado de manufactura. La alta frecuencia de lascas de reactivación registradas en el sitio La Peligrosa 2 (Salgán 2012), podrían apoyar la idea.

Por su parte, la obsidiana Cerro Huenul estaría ingresando a la región en forma de nódulos, facilitado quizás por el modo de yacencia en la fuente y su excelente calidad (Barberena et al. 2011). En los sectores Central y Sur se registraron en esta materia prima, un núcleo agotado y una alta frecuencia de lascas externas. En ambos sectores los índices de corteza son altos y los de intensidad de reducción bajos, lo que podría indicar un acceso directo a la fuente pero poco frecuente, dada su abundancia menor a la esperada. En los sitios más próximos del sector Sur $(30 \mathrm{~km})$, la misma se encuentra representada por desechos de talla correspondientes a las primeras etapas de manufactura. No obstante, su variación aumenta en el sector Central, donde se presentan todas las etapas de reducción. El instrumental se encuentra trabajado en su mayoría en forma bifacial (puntas de proyectil) y como artefactos con rastros complementarios.

En cuanto a Las Cargas, solo se presenta en el sector Central, donde estaría ingresando como instrumentos terminados, lascas internas y lascas de reactivación de filos, con valores de corteza nulos y de reducción altos. El Peceño y Desconocida-A se registran sólo en el sector Norte en muy baja frecuencia, como lascas internas. La alta frecuencia de lascas de reactivación de filo y microlascas presentes en los conjuntos del sitio La Peligrosa (Salgán 2012), permiten sostener de forma tentativa que estas materias primas estarían ingresando como artefactos terminados, sujetos a mantenimiento y quizás altamente conservados. Por último la fuente Desconocida, se presenta en los sectores Central y Sur, con mayor variabilidad en clases artefactuales en el sector Central, nulos valores de corteza y alto índice de reducción. Las tendencias tecnológicas sugieren que la obsidiana Laguna El Maule y Cerro Huenul responderían a un acceso directo en los sectores Central y Sur e indirecto en el Norte. La obsidiana Las Cargas, El Peceño y las variedades desconocidas en cambio responderían a un acceso indirecto.

\section{Discusión}

Las evidencias geoquímicas y tecnológicas permiten sostener que las distancias relativas entre las fuentes y los sitios arqueológicos no responden a las expectativas generales esperadas, debido a que en los sitios arqueológicos analizados las fuentes 
más próximas no están mejor representadas que las ubicadas a mayor distancia (como lo observado en el sector Sur en obsidiana Cerro Huenul y en el sector Norte en obsidiana El Peceño), la curva de caída o modelo cuantitativo de distribución espacial de las materias primas (sensu Renfrew 1977) en variables como frecuencia y tamaño no siguen el patrón esperado en el caso de las fuentes Laguna El Maule y Cerro Huenul. Situación que se replica en la intensidad de uso de las materias primas, las cuales no siguen una línea de reducción acorde con el aumento de la distancia entre los sitios arqueológicos y las fuentes. Respecto a las expectativas tecnológicas, se desacatan tres ejemplos que escapan al patrón esperado y que podrían ser explicados en términos biogeográficos. En primer lugar, es inesperada la mayor frecuencia de obsidiana de Laguna El Maule en el sector Norte, en relación a la fuente de El Peceño, dado que esta última es la más próxima, de disponibilidad anual y de fácil acceso. En un trabajo previo se planteó que la región muestra evidencias de ejes de circulación oeste-este por sobre los norte-sur (Cortegoso et al. 2012; Giesso et al. 2011). Las razones que explican esta tendencia aún no han sido discutidas, pero podrían estar relacionadas con la dirección predominante de los ríos de la macro región, la existencia de restricciones territoriales que limiten el acceso y circulación de obsidiana El Peceño o problemas de muestreo (Salgán et al. 2012).

Otro punto a destacar en términos de distribución contra-intuitiva de obsidiana en El Payén, es la representación que tiene la fuente Cerro Huenul dentro de los sectores analizados. Si bien es la fuente más próxima a los sectores Central y Sur, se halla en menor proporción que lo esperado y no supera en ninguno de los casos los valores de frecuencia de la obsidiana Laguna El Maule, localizada a una distancia hasta tres veces mayor y de disponibilidad estacional. Las características tecnológicas de los ejemplares de Cerro Huenul son coherentes con la proximidad de esta fuente a la región, aunque no su abundancia en los conjuntos. Barberena et al. (2011) plantean la posibilidad de que el río Colorado funcione como una barrera temporal (sensu Veth 1993) durante periodos específicos del año. En el tiempo, esto habría contribuido a una baja representación macro regional de la cantera. Si se acepta el criterio de Barberena et al. (2011), la mencionada cuenca también limitaría el acceso estival a las fuentes cordilleranas, lo que es incongruente con el registro de artefactos procedentes de Laguna El Maule.

Por último, el tercer punto que no condice con las expectativas de distancia entre sitios y fuentes, ocurre en relación a la fuente de Laguna El Maule. Hay una menor frecuencia de obsidiana procedente de Laguna El Maule en el sector Central en relación al Sur, y a su vez las etapas de manufactura más frecuentes entre los artefactos recuperados en el sector Central corresponden a las últimas etapas de manufactura. Esto es contrario a lo esperado, dado que el área Central se encuentra más próxima a la fuente Laguna El Maule que el sector Sur. Todos los indicadores mencionados parecen mostrar que la obsidiana estaría ingresando al sector Central desde el sector Sur, facilitando su circulación por los ríos Barrancas y Colorado (recorrido de traslado aproximado de $270 \mathrm{~km}$ ) (Figura 3, ruta A) y desde este último al Norte. Esta errática circulación de la obsidiana puede ser explicada por la presencia de las Sierras de Reyes y de Cara-Cura, ubicadas al oeste de los sectores Central y Sur. Este cordón serrano sólo posee un paso en el centro norte a través del área de Los Volcanes (camino más corto de ca. $200 \mathrm{~km}$, pero sin disponibilidad de agua) (Figura 3, ruta B). Una vía alternativa de circulación puede darse siguiendo el curso del río Grande (evitando el cordón serrano de Reyes y de Cara Cura; ca. $350 \mathrm{~km}$ ) que se desvía hacia el sur, antes de ingresar a las áreas bajo estudio (Figura 3, ruta C). 


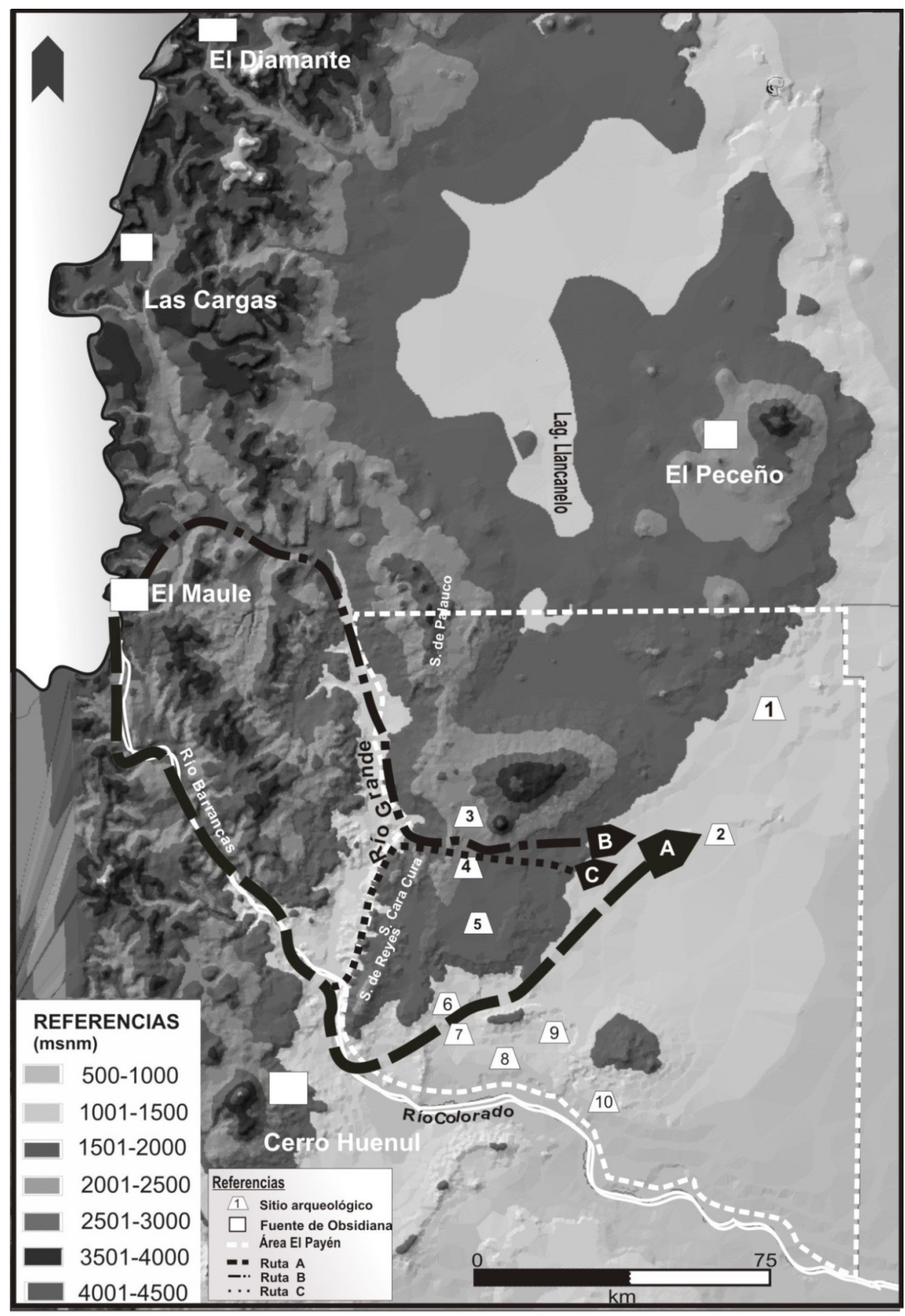

Figura 3. Rutas propuestas de circulación de obsidiana de mayor frecuencia en área El Payén. Referencia: Muestreos de obsidiana, 1. La Corredera; 2. La Peligrosa 2; 3. Puesto Mira; 4. Loma Alta 5. ALPA Este; 6. Agua de Pérez; 7. Carmonina 1; 8. PRC; 9. Rincón Blanco; 10. PRC Tren. 
En una escala macro regional, se ha registrado obsidiana Laguna El Maule y Las Cargas en el valle medio del río Grande, en conjuntos atribuidos al Holoceno temprano (entre 8.000 y 7.000 años AP) y a la primera mitad del Holoceno tardío (entre 4.000 y 2.000 años AP) (Durán 2000; Neme et al. 2011). Asimismo, en este último bloque temporal se incorpora en los registros arqueológicos la obsidiana procedente de Cerro Huenul (Neme et al. 2011). Estos pueden ser considerados indicadores de direccionalidad de transporte de este recurso y posibles vías interacción con La Payunia (Salgán 2012).

En el oeste de la región pampeana, Giesso et al. (2008) identificaron artefactos procedentes de Laguna El Maule y Cerro Huenul en contextos asociados a la primera mitad del Holoceno tardío. Los autores sostienen que el intercambio de bienes sería frecuente a través de las grandes cuencas hídricas, tales como los ríos Colorado, Barrancas, Grande y Neuquén (sur de Mendoza y norte Neuquén) y, Chadileuvú y Curacó (La Pampa) (Giesso et al. 2008; Berón 2010). En este marco, las vías de circulación y transporte de obsidiana propuesta en la figura 3 (rutas B y C) cobran relevancia en el área El Payén, al tiempo que darían cuenta de la presencia de obsidiana Las Cargas, sólo en el sector Central.

\section{Conclusión}

El abordaje tecnológico de la distribución de artefactos de obsidiana de procedencia conocida, ha permitido generar tendencias referentes a los modos de aprovisionamiento, rango de acción, posible direccionalidad de interacción entre poblaciones y patrones de distribución contra intuitivos. Tendencias difíciles de enfocar sólo desde los estudios geoquímicos. Se destaca la potencialidad del análisis tecnológico y en particular de los indicadores de secuencias de producción e intensidad de uso de las materias primas, para discutir con mayor profundidad la amplitud del rango de acción de las poblaciones humanas. Las investigaciones subsiguientes y el muestreo intensivo de la región, permitirán ajustar y contrastar los postulados aquí presentados.

Agradecimientos: Agradecemos las acertadas observaciones que nos realizaran dos evaluadores anónimos de este trabajo. También a Michael D. Glascock, Martín Giesso, Victor Durán y Valeria Cortegosos con quienes encaramos los estudios de caracterización geoquímica y con quienes frecuentemente discutimos sus interpretaciones. Este trabajo se enmarca en el PICT 2012-1015 y el Programa de UNCuyo "Perspectivas Paleoecológicas para el Estudio de las relaciones Humano ambientales en el Centro Occidente Argentino". Ninguno de ellos es responsable de los errores u omisiones que se incluyan aquí.

\section{Bibliografía citada}

Aschero, C.

1975 Ensayo para una clasificación morfológica de artefactos líticos aplicados a estudios tipológicos comparativos. Informe a CONICET. Ms.

1983 Ensayo para una clasificación morfológica de artefactos líticos aplicada a estudios tecnológicos comparativos. Apéndice A-C. Revisión 1983. Cátedra de Ergología y Tecnología. Facultad de Filosofía y Letras, UBA. Ms. 
Aschero C. y S. Hocsman

2004 Revisando cuestiones tipológicas en torno a la clasificación de artefactos bifaciales. Temas de Arqueología, Análisis Lítico (ed. A. Acosta, D. Loponte y M. Ramos), pp. 7-26. Buenos Aires.

Bamforth, D. B.

1991 Technological organization and hunter-gatherer land use. American Antiquity 56: 216235.

Barberena, R., A. Hajduk; A.F. Gil, G. A. Neme, V.A. Durán, M.D. Glascock, M. Giesso, K. Borrazzo M.P. Pompei, M.L. Salgán, V. Cortegoso, G. Villarosa y A.A. Rughini

2011 Obsidians in the south-central Andes: geological, geochemical, and archaeological assessment of north Patagonian sources (Argentina).Quaternary International 245(1): 25-36.

Beck, C., A.K. Taylor, G.T. Jones, C.M. Fadem, C.R. Cook y S.A. Millward

2002 Rocks are heavy: transport costs and Paleoarchaic quarry behaviour in the Great Basin. Journal of Anthropological Archaeology 21: 481-507.

Berón, M.A.

2010 Circuitos regionales y conflictos intergrupales prehispánicos. Evidencias arqueológicas de violencia y guerra en La Pampa Occidental Argentina. Actas del XVII Congreso Nacional de Arqueología Chilena I: 493-502.

Binford, L. R.

1979 Organization and formation processes: looking at curated technologies. Journal of Anthropological Research 35 (3): 255-273.

Charlin, J.

2007 Una perspectiva espacial de la intensidad de uso de las materias primas líticas en el campo volcánico Pali Aike (Prov. Santa Cruz, Argentina). Arqueología de Fuego-Patagonia. Levantando piedras, desenterrando huesos... y develando arcanos (ed., por F., M. Carballo, A. Martinic, Prieto, y G. Bahamonde), pp. 129-150. CEQUA. Punta Arenas, Chile.

2009 Aprovisionamiento, explotación y circulación de obsidianas durante el Holoceno tardío en Pali Aike (Provincia de Santa Cruz). Relaciones de la Sociedad Argentina de Antropología XXXIV: 53-73.

Civalero, M.T. y N. Franco

2003 Early Human Occupations in Western Santa Cruz Province, Southernmost South America. Quaternary International 109-110: 77-86.

Cortegoso V., G. Neme, M. Giesso, V. Durán y A. Gil

2012 El uso de la obsidiana en el sur de Mendoza. Paleoecología humana en el sur de Mendoza (ed. por A. Gil y G. Neme), pp. 180-211. Sociedad Argentina de Antropología.

De Francesco, A., V. Durán, A. Bloise y G. Neme.

2006 Caracterización y procedencia de obsidianas de sitios arqueológicos del área natural protegida Laguna del Diamante (Mendoza, Argentina) con metodología no destructiva por fluorescencia de rayos (XRF). Anales de Arqueología y Etnología 61: 53-67.

Durán, V.

2000 Poblaciones indígenas de Malargüe. Su arqueología e historia. CEIDER Nº 1, Mendoza. 
Durán, V., M. Giesso, M. Glascock, G. Neme, A. Gil y L. Sanhueza

2004 Estudios de fuentes de aprovisionamiento y redes de distribución de obsidiana durante el Holoceno Tardío en el sur de Mendoza (Argentina). Estudios Atacameños 28: 25-43.

Ericson, J. E.

1984 Toward the analysis of lithic production system. Prehistoric Quarries and Lithic Production (ed. por J. E. Ericson y B. Purdy), pp. 1-9. Cambridge University Press.

Franco, V. N.

2004 La organización tecnológica y el uso de las escalas espaciales amplias. El caso del sur y oeste de Lago Argentino. Temas de Arqueología, Análisis Lítico (ed. por A. Acosta, D. Loponte y M. Ramos), pp. 101-144, Buenos Aires.

Giesso, M. y M. Berón

2008 Obsidian in Wester Pampas, Argentina: source characterization and provisioning strategies. LAOS Bulletin 38: 15-18.

Giesso, M., V. Durán, G.A. Neme, M.D. Glascock, V. Cortegoso, A.F. Gil y L. Sanhueza 2011 Application of a Portable XRF to Study Obsidian Use in the Central Andes region of Argentina and Chile. Archaeometry 53 (1): 1-21.

Glascock, M. D.

2010 Comparison and contrast between XRF and NAA: Used for characterization of obsidian sources in Central Mexico. X Ray Fluorescence Spectrometry (XRF) in Geoarchaeology (ed. por M.S. Shackley), pp. 161-192. Springer, NewYork.

Gould, R. y S. Saggers

1985 Lithic procurement in Central Australia: a closer look at Binford's idea of embeddedness in archaeology. American Antiquity 50 (1): 117-136.

Ingbar, E.

1994 Lithic Material Selection and Technological Organization. The organization of North American prehistoric chipped stone Technologies (ed. por P. Carr), pp. 45-56. Archaeological Series 7, Michigan.

Ingbar, E., M. Larson y B. Bradley 1989 A Nontypological Approach to Debitage Analisis. Experiments in Lithic Technology (ed. por D. S. Amick y R. P. Mauldin), pp. 67-99. BAR International Series 528, Oxford.

Jones G.T, L. M. Fontes, R.A. Horowitz, C. Beck, y D.G. Bailey

2012 Reconsidering Paleoarchaic Mobility in the Central Great Basin . American Antiquity 77 (2): 351-368.

Kuhn, S.

1989 Hunter-gatherer foraging organization and strategies of artifact replacement and discard. Experiments in Lithic Technology (ed. por D. S. Amick y R. P. Mauldin), pp. 33-47. BAR International Series 528, Oxford.

2004. Upper Paleolithic raw material economies at Ücagizli cave, Tuckey. Jounal of Anthropological Archaeology 23: 431-448. 
Meltzer, D.

1989 Was Stone Exchange Among Eastern North American Paleoindians?. Eastern Paleoindian lithic resource use (ed. por C. Ellis y J. Lothrop), pp. 11-39. Westview Press.

Neme, G. y A. Gil

2008 Biogeografía humana en los andes meridionales: Tendencias arqueológicas en el sur de Mendoza. Chungará 40 (1): 5-18.

Neme, G.A., A.F. Gil, R. Garvey, C. Llano, A. Zangrando, F. Franchetti, C. De Francesco y C.T. Michelli

2011 El registro arqueológico de la Gruta de El Manzano y sus implicancias para la arqueología de nordpatagonia. Magallania 39(2): 243-265.

Renfrew, C.

1977 Alternative models for exchange and spatial distribution. Exchange Systems in Prehistory (ed. por T. Earle y J. Ericson), pp. 71-90. Academic Press, New York.

Salgán, M.L.

2012 Organización tecnológica y biogeografía humana en La Payunia, sur de la Provincia de Mendoza. Tesis Doctoral inédita, Facultad de Ciencias Naturales y Museo, Universidad Nacional de La Plata.

Salgán, M.L., A. Gil y G. Neme

2012 Obsidianas en La Payunia (sur de Mendoza, Argentina): patrones de distribución e implicancias en la ocupación regional. Magallania 40 (1): 263-277.

Salgán, M.L., G.W. Bertotto y M.M. Garrido

2013 Petrografía y procedencia de rocas silíceas en La Payunia (Malargüe, provincia de Mendoza). Intersecciones en Antropología. En Prensa

Seelenfreund, A., C. Rees, R. Bird, G. Bailey, R. Bárcena y V. Durán

1996 Trace-Element Analysis of Obsidian Sources and Artifacts of Central Chile (Maule

River Basin) and Western Argentina (Colorado River). Latin American Antiquity 7(1): 7-20.

Stern, C. y N. Franco

2000 Obsidiana gris verdosa veteada en la cuenca superior del río Santa Cruz, extremo sur de Patagonia. Anales del Instituto de la Patagonia, Serie Ciencias Humanas 28: 265-273.

Veth. P. M.

1993 Islands in the Interior. The Dynamics of Prehistoric Adaptations within the Arid Zone of Australia. International Monographs in Prehistory, Archaeological Series 3. 